\title{
Highlights
}

Integrated Material and Process Evaluation of Metal-organic Frameworks Database for Energy-efficient $\mathrm{SF}_{6} / \mathrm{N}_{2}$ Separation

Jaehoon Cha, Seongbin Ga, Seung-jun Lee, Soomyung Nam, Youn-Sang Bae, Yongchul G. Chung

- Multi-scale screening to find the best MOFs for the adsorptive separation of $\mathrm{SF}_{6}$

- GCMC simulations of 14,000 MOFs from CoRE MOF 2019 database

- Process-level evaluation of MOFs in VSA/PSA processes

- Comparison of top ranked MOFs of VSA/PSA in different pump efficiency scenarios 


\section{Integrated Material and Process Evaluation of Metal-organic Frameworks Database for Energy-efficient $\mathrm{SF}_{6} / \mathrm{N}_{2}$ Separation}

Jaehoon Cha ${ }^{\mathrm{a}}$, Seongbin Ga ${ }^{\mathrm{a}}$, Seung-jun Lee ${ }^{\mathrm{b}}$, Soomyung Nam ${ }^{\mathrm{a}}$, Youn-Sang Bae $^{\mathrm{b}, *}$, Yongchul G. Chung ${ }^{\mathrm{a}, * *}$

${ }^{a}$ School of Chemical Engineering, Pusan National University Busan, 46241, Korea (South)

${ }^{b}$ Department of Chemical and Biomolecular Engineering Seoul, 03722, Korea (South)

\section{Abstract}

In this work, we proposed multi-scale screening, which employs both molecular and process-level models, to identify high-performing MOFs for energyefficient separation of $\mathrm{SF}_{6}$ from $\mathrm{SF}_{6}$ and $\mathrm{N}_{2}$ mixture. Grand canonical Monte Carlo (GCMC) simulations were combined with ideal adsorption process simulation to computationally screen 14,000 metal-organic frameworks (MOFs) for adsorptive separation of $\mathrm{SF}_{6} \quad \mathrm{~N}_{2}$. More than 150 high-performing MOFs were identified based on the results from GCMC simulations at the pressure and vacuum swing conditions, and subsequently evaluated using the ideal adsorption process simulation. High-performing MOFs selected for the VSA conditions are able to achieve the $90 \%$ target purity level of $\mathrm{SF}_{6}$ but none of the selected MOFs for PSA conditions could. Cascade PSA configuration was proposed and adopted to improve the purity level of the separated $\mathrm{SF}_{6}$.

*mowbae@yonsei.ac.kr

**drygchung@gmail.com 
Cascade PSA configuration was also adopted to improve the purity. In the pump efficiency scenarios of 80,20 , and $10 \%$, the VSA and cascade PSA cases were compared, which concluded $10 \%$ scenario prefers the PSA case whereas the VSA case is favored in the others. Top-performing MOFs identified from the multi-scale computational approach were found to be able to produce $90 \%$ purity $\mathrm{SF}_{6}$ with $0.10-0.4$ and $0.5-1.4 \mathrm{MJ}$ per $\mathrm{kg}$ of $\mathrm{SF}_{6}$ for VSA and PSA, respectively.

Keywords: $\mathrm{SF}_{6}$, High-throughput Screening, Adsorption process

\section{Introduction}

As we transition from centralized power systems (such as coal-fired power plants) to more decentralized ones (such as solar and wind) to power the planet, it becomes critical to reliably and securely distribute the electricity to where it demands. The central to the electricity supply chain is the switchgear, and a recent report indicates that the market for the highperforming switchgear is expected to reach 152 billion dollar by 2029 [1]. The most effective form of the switchgear is the gas-insulated switchgear (GIS), where the conductors and contacts are insulated by pressurized dielectric gas, such as sulfur hexafluoride $\left(\mathrm{SF}_{6}\right)$. The high dielectric strength of $\mathrm{SF}_{6}$ makes the gas useful in GIS, where $\mathrm{SF}_{6}$ is typically mixed with relatively cheap $\mathrm{N}_{2}$. However, $\mathrm{SF}_{6}$ has been identified as one of the main greenhouse gases in the Paris Agreement 2] due to its high global warming potential (GWP: 22,800 23,900). According to a report Simmonds et al. [3], the global $\mathrm{SF}_{6}$ emission 
was increased by roughly $260 \%$ from 1978 to 2018, which emphasizes the severity of the problem. Because of this, the development of advanced materials and separation processes that can selectively and efficiently capture the $\mathrm{SF}_{6}$ from $\mathrm{SF}_{6} / \mathrm{N}_{2}$ mixtures is necessary to reduce the emission of $\mathrm{SF}_{6}$ from the distributed electrical transmission systems.

Recently, a number of advanced materials and separation processes, such as adsorption-based and membranes-based methods [4, 5, 6, have been proposed in the literature as a promising method to perform the $\mathrm{SF}_{6} / \mathrm{N}_{2}$ separation. For the adsorption-based separation process, there are two representative modes of operation: 1) vacuum swing adsorption (VSA), where the gas is adsorbed at the feed pressure and desorbed below the pressure, and 2) pressure swing adsorption (PSA), where the compressed gas is adsorbed and desorbed at the lower pressure. The key performance indicators (KPIs), such as product purity and recovery, for these two modes of operation heavily depend on the choice of the adsorbent material, which necessitates the development of high-performing adsorbent materials, such as metal-organic frameworks.

Metal-organic frameworks (MOFs) are a class of nanoporous materials that find their application in modern engineering applications, such as gas storage and separation, catalysis, and chemical sensing [7, 8, 9]. Several MOFs have been reported in the literature for $\mathrm{SF}_{6} / \mathrm{N}_{2}$ separation application [10, 11, 12]. For example, Senkovska and coworkers have reported HKUST-1 showed the best performance in $\mathrm{SF}_{6}$ separation $\left(\mathrm{SF}_{6}\right.$ uptake $120 \mathrm{~cm}^{3} / \mathrm{g}$ at 18 
bar), whereas MIL-101 ( $\mathrm{SF}_{6}$ uptake $276 \mathrm{~cm}^{3} / \mathrm{g}$ at $\left.18 \mathrm{bar}\right)$ and DUT-9 (SF 6 uptake $275 \mathrm{~cm}^{3} / \mathrm{g}$ at $18 \mathrm{bar}$ ) had the highest storage capacities. Another study by Chuah and coworkers have reported modified hierarchically structured HKUST-1 with both very high $\mathrm{SF}_{6}$ uptake $\left(4.98 \mathrm{mmol} \cdot \mathrm{g}^{-} 1\right.$ at $25^{\circ} \mathrm{C}$ and 1 bar) and $\mathrm{SF}_{6} / \mathrm{N}_{2}$ selectivity (around 70 at $25^{\circ} \mathrm{C}$ ). Kim and coworkers have reported a zirconium-based $\mathrm{MOF}$, UiO-67, with high $\mathrm{SF}_{6} / \mathrm{N}_{2}$ selectivity (30 - 37 at 10 bar). Nevertheless, the number of MOFs evaluated for this application is limited, and their full potential as part of the process modeling has not been evaluated in the literature.

Toward this end, computational high-throughput screening combined with multi-scale modeling could be used to quickly identify high-performing MOFs from large material databases [13, 14]. Computational screening has been applied to discover high-performing MOFs for energy storage applications, such as methane [15] and hydrogen storage [16, 17, 18], $\mathrm{CO}_{2}$ capture [7], isomer separation [19], among others [20]. In this work, we carried out high-throughput, multi-scale computational screening of CoRE MOF 2019 database [21] to identify the high-performing MOFs for the $\mathrm{SF}_{6} / \mathrm{N}_{2}$ separation. The data obtained from the molecular-level simulations were directly used for the process-level simulation to evaluate the process-level performance indicators, such as the product purity, recovery, and the energy consumption of the process per mole of produced $\mathrm{SF}_{6}$, of MOFs for VSA and PSA operations. Moreover, we explored ways to increase the product purity of PSA operation by introducing a cascade PSA configuration to achieve $90 \%$ purity 
level of produced $\mathrm{SF}_{6}$. Our multi-scale screening study was able to identify top-performing MOFs for $\mathrm{SF}_{6} / \mathrm{N}_{2}$ separation for VSA and PSA conditions. We found the MOFs that are optimal for VSA and PSA operations have different physical and chemical properties. Energy consumption analyses show that the VSA operation was able to produce $90 \%$ pure $\mathrm{SF}_{6}$ with $0.1-0.4$ MJ per kg of $\mathrm{SF}_{6}$ for $74 \mathrm{MOFs}$ (out of $87 \mathrm{MOFs}$ ). None of the MOFs identified from high-throughput screening (using selectivity and working capacity metrics) for PSA operation were able to meet the $90 \%$ pure $\mathrm{SF}_{6}$. Instead, we were able to meet the $90 \% \mathrm{SF}_{6}$ purity level using the cascade PSA configuration, which uses two PSA trains in series. Cascade PSA was able to produce $90 \%$ pure $\mathrm{SF}_{6}$ with 0.5 - $1.4 \mathrm{MJ}$ per $\mathrm{kg}$ of $\mathrm{SF}_{6}$ for $36 \mathrm{MOF}$ (out of 74 MOFs). Further analyses showed that the cascade PSA operation could be more energy efficient mode of operation than VSA operation depending on the efficiency of vacuum pump used for the operation. While none of the MOFs that emerged as the top-performing MOFs for VSA process from this work were synthesized and tested to date, UiO-67, UiO-66 and HKUST-1, which have been previously tested for the $\mathrm{SF}_{6} / \mathrm{N}_{2}$ separation application for both VSA and PSA conditions, were identified as the top-performing MOFs to perform the separation using cascade PSA. 

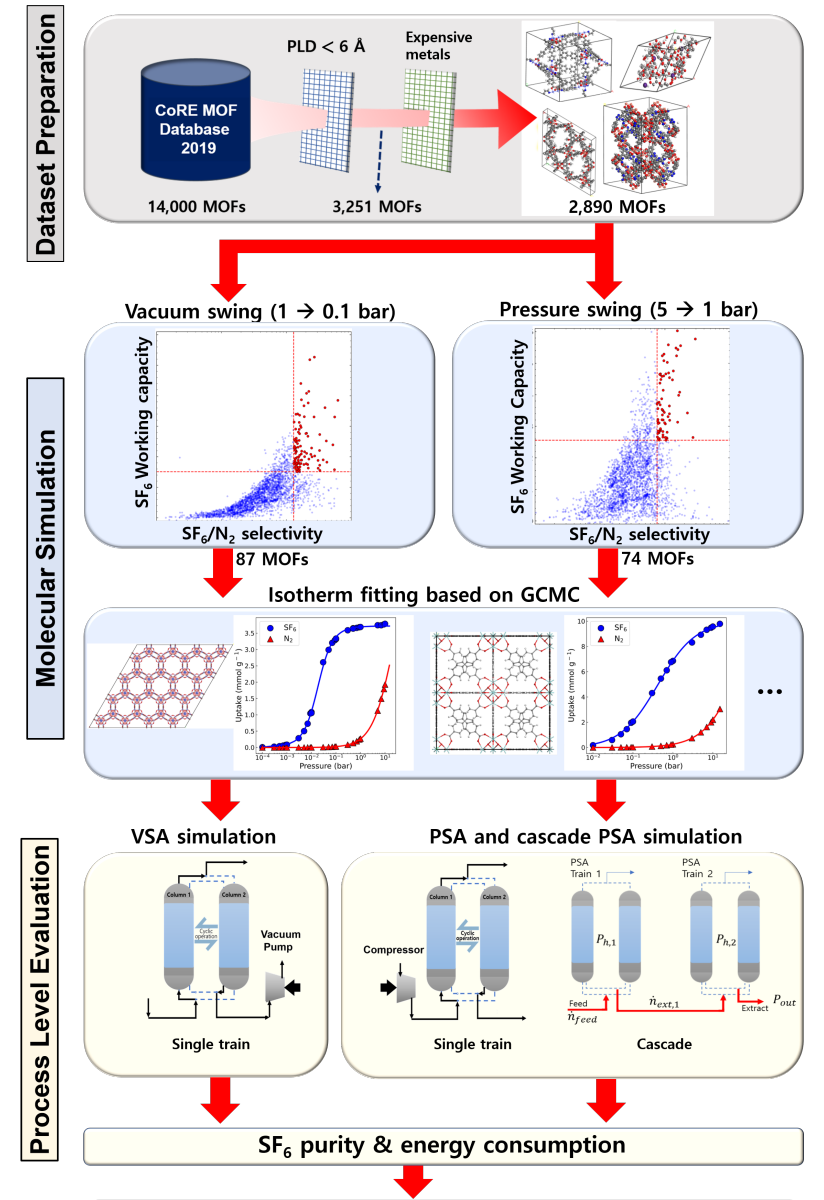

High performing MOFs for $\mathrm{SF}_{6} / \mathrm{N}_{2}$ separation

Figure 1: Computational workflow of high-throughput, multi-scale computational screening procedure employed in this work. 


\section{Computational Methods and Models}

\subsection{CoRE MOF database and Filtering Criteria}

CIF files for MOFs and their physical properties were collected from CoRE MOF 2019 database[21]. To reduce the computational resources used for the screening, we first removed MOFs with the pore limiting diameter (PLD) smaller than $6 \AA$ (which is slightly larger than the diameter of $\mathrm{SF}_{6}$ model $(5.6 \AA)$ used in this work). We also removed MOFs with precious metals (see the Supplementary Information Table S1). Based on these two filtering criteria, we arrived at 2,890 MOFs from 14,000 MOFs from the CoRE MOF 2019 database (Figure 1). We labeled each of 2,890 MOFs with a screening ID, and the screening ID is used throughout this work to identify a MOF. The high-throughput screening data and physical properties of all 2,890 MOFs are provided as a part of the Supplementary Information (HTS-result.csv).

\subsection{Grand Canonical Monte Carlo (GCMC) Simulation}

GCMC simulations were carried out to evaluate the $\mathrm{SF}_{6}$ and $\mathrm{N}_{2}$ adsorption properties of 2,890 MOFs using RASPA 2.0[22]. High-throughput GCMC simulations were carried out at fixed temperature $298 \mathrm{~K}$ with 1:9 $\mathrm{SF}_{6}$ and $\mathrm{N}_{2}$ mixture composition. For high-throughput screening, the $\mathrm{SF}_{6}$ and $\mathrm{N}_{2}$ mixture uptakes were calculated at three different pressure points -0.1 bar, 1 bar and 5 bar. A GCMC simulation consists of 10,000 cycles for equilibration and 20,000 cycles for the ensemble averages, where a single cycle is 
equal to the number of adsorbate molecule in the system or 20 (whichever is greater). Translation, rotation, reinsertion and swap Monte Carlo moves were used with equal probabilities for sampling.

$\mathrm{SF}_{6}$ (or $\mathrm{N}_{2}$ ) working capacity and $\mathrm{SF}_{6} \mathrm{~N}_{2}$ selectivity were calculated based on the GCMC simulation results. The working capacity, WC, was computed by taking the difference between the $\mathrm{SF}_{6}$ or $\mathrm{N}_{2}$ uptakes at the adsorption (1 bar for VSA and 5 bar for PSA) and desorption (0.1 bar for VSA and 1 bar for PSA) pressures (1):

$$
W C=N_{a d s}-N_{d e s}
$$

Here, $N_{a d s}$ and $N_{\text {des }}$ are the molecule loadings at the adsorption and desorption conditions. The selectivity between $\mathrm{SF}_{6}$ and $\mathrm{N}_{2},\left(\alpha_{S F_{6} / N_{2}}\right)$ at the adsorption conditions (1 bar for VSA and 5 bar for PSA) was calculated using the following Eq. (2):

$$
\alpha_{S F_{6} / N_{2}}=\frac{q_{S F_{6}} / q_{N_{2}}}{p_{S F_{6}} / p_{N_{2}}}
$$

Here, $q_{S F_{6}}$ and $q_{N_{2}}$ are the uptake $(\mathrm{mmol} / \mathrm{g})$ of $\mathrm{SF}_{6}$ and $\mathrm{N}_{2}$, and $p_{S F_{6}}$ and $p_{N_{2}}$ are the partial pressure of $\mathrm{SF}_{6}$ and $\mathrm{N}_{2}$, respectively.

\subsection{Force fields}

The interaction between atoms in the systems were approximated with the van der Waals (vdW) interaction, which was modeled with the LennardJones (LJ) 12-6 type Equation (Eq. 3). The interaction parameters between 
different atom types were approximated using the Lorentz-Berthelot mixing rules. A vdW cutoff distance of 12.8 Åwas used to truncate the vdW interactions between atoms.

$$
U_{i j}=4 \varepsilon_{i j}\left[\left(\frac{\sigma_{i j}}{r_{i j}}\right)^{12}-\left(\frac{\sigma_{i j}}{r_{i j}}\right)^{6}\right]
$$

Here, $U_{i j}$ is the interaction energy between atoms $i$ and $j, r_{i j}$ is the center-to-center distance between the two atoms. The LJ parameters for all framework atoms were taken from the DREIDING force field[23]. If the atom types were not available in DREIDING force field, we have used the UFF force field [24]. The $\mathrm{SF}_{6}$ molecule was modelled using a 7 -site model from the work of J. Samios and coworkers [25]. The LJ parameter of $\mathrm{N}_{2}$ was taken from the Transferable Potential for Phase Equilibria (TraPPE) force field 26]. All the LJ parameters are listed in the Table S2 and Table S3 of Supplementary Information.

\subsection{Widom Particle Insertion}

The heat of adsorption of $\mathrm{SF}_{6}$ and $\mathrm{N}_{2}$ for all MOFs were calculated using the Widomś particle insertion method [27] as implemented in RASPA 2.0. 


\subsection{Ideal Adsorption Process Simulation}

We adopted an ideal adsorption process formalism, which has been re-

cently proposed [28, 29], to quickly evaluate the upper limit performance of MOFs. The ideal adsorption process assumes optimal operation of the adsorption process so that the full potential of each MOF can be evaluated. Some key assumptions for the ideal adsorption process are:

- Discrete pressure swing: No loss in $\mathrm{SF}_{6}$ purity and energy due to pressure gradient both across the cycle time and across the column, which leads to two operation steps (adsorption and desorption) for a single cycle.

- Fast mass-transfer: $100 \%$ purity of raffinate (weakly adsorbing component stream) due to fast mass-transfer between bulk gas phase to adsorbed phase.

- Isothermal operation: Heat generated from adsorption is neglected.

Figure 2 shows the cyclic operation of VSA and PSA process configurations that we considered in this work. For the VSA, a vacuum pump is located at the end of the process while for the PSA, a compressor is located at the beginning of the process configuration. Figure 2 (a) and (b) show the VSA and PSA operations where $\mathrm{N}_{2}$ is produced as the raffinate stream, and the product $\mathrm{SF}_{6}$ is produced as the extract stream. Note that $\mathrm{N}_{2}$ component is also present in the extract stream as well since some $\mathrm{N}_{2}$ molecules are 

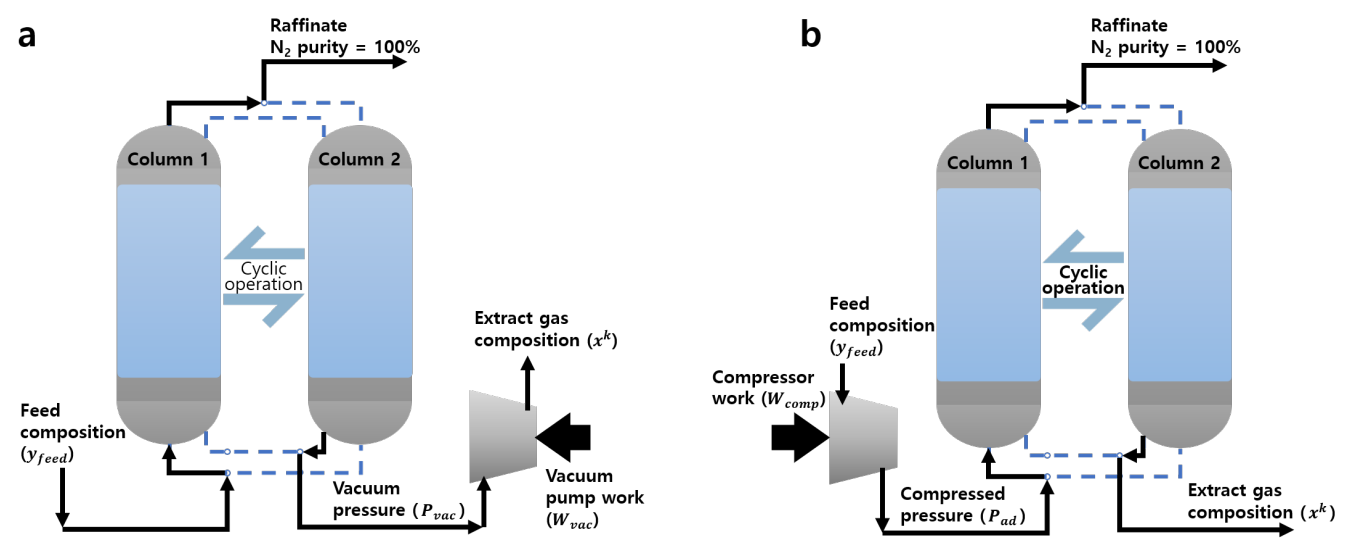

Figure 2: (a) Ideal VSA and (b) Ideal PSA progress diagram and key state variables

adsorbed onto the MOFs along with $\mathrm{SF}_{6}$. In both VSA and PSA operations, two columns operate asynchronously in which the adsorption/desorption operations are occurring at the same time, and the cyclic steady-states (CSS) in both columns are assumed for both columns as part of the ideal adsorption process consideration. For both operations, two columns filled with the same MOFs are used for the simulation. During the adsorption period, all $\mathrm{SF}_{6}$ and small amount of $\mathrm{N}_{2}$ are trapped in the column while pure $\mathrm{N}_{2}$ is produced as the raffinate. This is because $\mathrm{SF}_{6}$ is more strongly adsorbing component than $\mathrm{N}_{2}$. During the desorption period, the trapped $\mathrm{SF}_{6}$ and $\mathrm{N}_{2}$ desorb from the MOFs and exit the column as the extract.

The mixture adsorption isotherm data at different bulk phase gas compositions are necessary to evaluate the performance of MOFs at the processlevel. Single-component GCMC simulations were carried out for $\mathrm{SF}_{6}$ and $\mathrm{N}_{2}$ from 0.01 bar to 10 bar at $298 \mathrm{~K}$. All the calculated GCMC isotherm data 
points were fitted using open-source python code pyIAST [30] with following isotherm models expressed with Eq. (4) and Eq. (5):

$$
\begin{gathered}
\text { Langmuir }: q=M \frac{K p}{1+K p} \\
\text { Quadratic }: q=M \frac{\left(K_{a}+2 K_{b} p\right) p}{\left(1+K_{a} p+K_{b} p^{2}\right)}
\end{gathered}
$$

Here, $q$ is the uptake and $p$ is the pressure of the system. $(M, K)$ are the isotherm parameters for the Langmuir model and $\left(M, K_{a}, K_{b}\right)$ are for the quadratic model. The isotherm models and parameters of $\mathrm{SF}_{6}$ and $\mathrm{N}_{2}$ were combined with ideal adsorbed solution theory (IAST) to model the mixture adsorption uptake of a MOF at different bulk phase gas composition[31]. Between the two models, a model with the smaller root mean square error (RMSE) was selected for the process-level simulation. All the fitted parameters are provided as part of the the Supplementary Information.

\subsection{Derivation of Product Purity and Sensitivity Analyses}

To evaluate the process-level performance of a $\mathrm{MOF}$, we used $\mathrm{SF}_{6}$ purity and the energy consumption per unit $\mathrm{SF}_{6}$ as the key performance indicators (KPIs). Using the ideal adsorption process formalism, we derive the composition of the extract to determine the $\mathrm{SF}_{6}$ purity for a given MOF $k$ ( $\boldsymbol{x}$ in Figure 2). For simplicity, we omitted the superscript $k$ throughout the section for clarify. 
In the bulk gas phase with $n$ components, the mole fractions of each gas component $i$ is represented by an element of $\boldsymbol{x}$ vector:

$$
\boldsymbol{x} \equiv\left(x_{1}, x_{2}, \ldots, x_{n}\right)
$$

For the binary component of $\mathrm{SF}_{6}$ and $\mathrm{N}_{2}$ mixture, we could further simplify the above expression in terms of the mole fraction of $\mathrm{SF}_{6}\left(x_{1}\right)$ :

$$
\boldsymbol{x}=\left(x_{1}, 1-x_{1}\right)
$$

where, $x_{1}$ is the bulk gas phase mole fraction of $\mathrm{SF}_{6}$, which is equivalent to the $\mathrm{SF}_{6}$ composition of the extract stream (i.e., $\mathrm{SF}_{6}$ purity).

The bulk gas phase mole fractions of all components can be determined based on the feed and operating conditions. Feed conditions include feed composition and temperature $\left(y_{\text {feed }}, T_{\text {feed }}\right)$, and the operating condition is the pressure the adsorption column undergoes.

In both ideal VSA and PSA operations, the system goes through the pressure swing between $P_{h}$ and $P_{l}$ while the other operating conditions, such as the feed velocity and step time, are assumed to be optimized at the fixed pressure swing range. In the VSA process, the vacuum pressure $\left(P_{v a c}\right)$ created by the vacuum pump is used for the low pressure $\left(P_{l}\right)$ while 1 bar is used for the high pressure $\left(P_{h}\right)$. For the PSA process, the adsorption pressure $\left(P_{a d s}\right)$ made by the compression of the feed flow is used for the high pressure $\left(P_{h}\right)$ while the 1 bar is used for the low pressure $\left(P_{l}\right)$. 
The gas uptakes at adsorption/desorption conditions are based on the mixture isotherm function, $f_{\text {iso }, i}(\cdot)$.

$$
\begin{aligned}
& q_{a d s, i}=f_{\text {iso }, i}\left(y_{\text {feed }}, P_{h}, T_{\text {feed }}\right) \\
& q_{\text {des }, i}=f_{i s o, i}\left(\boldsymbol{x}, P_{l}, T_{\text {feed }}\right)
\end{aligned}
$$

For the mixture isotherm, the IAST calculations of $i$ component $\left(f_{i s o, i}\right)$ were carried out based on the following equations :

$$
\begin{gathered}
\pi_{i}\left(P_{i}^{o}\right) \equiv R T \int_{P}^{P_{i}^{o}} \frac{f_{i}^{o}(P)}{P} d P \\
x_{i}^{\text {solid }} \times P_{i}^{o}=P_{i} \\
\pi_{1}\left(P_{1}^{o}\right)=\pi_{2}\left(P_{2}^{o}\right) \\
\frac{1}{q_{\text {tot }}}=\sum_{i=1}^{2} \frac{x_{i}^{\text {solid }}}{f_{i}^{o}\left(P_{i}^{o}\right)} \\
q_{i}=q_{\text {tot }} \times x_{i}^{\text {solid }}
\end{gathered}
$$

Using the definition of spreading pressure $\left(\pi_{i}^{o}\right)$ of Eq. (10), all the mole fractions of component $i$ could be obtained via Eq. (11) and Eq. (12), and the obtained values lead to the uptake values with Eq. (13) and Eq. (14). These equations of IAST convert the pure isotherm models $\left(f_{i s o, i}^{o}\right)$ into the mixture isotherm model $\left(f_{i s o, i}\right)$, which indicates the procedure to find the uptake $\left(q_{i}\right)$ satisfying the above equations. During the procedure, 
the recursive computations are required for solid phase mole fraction $\left(x_{i}^{\text {solid }}\right)$ satisfying both Eq. (11) and Eq. (12). In the procedure, the hypothetical pressure, partial pressure of each component, and total uptake are expressed with $P_{i}^{o}, P_{i}$ and $q_{t o t}$. In the above equations, $\mathrm{R}$ and $\mathrm{T}$ indicate gas constant and temperature.

Note that during the adsorption period, the uptake in a MOF is defined as a function of feed flow mole fraction $\left(y_{f e e d}\right)$ while the gas uptake at the desorption period is a function of bulk gas phase mole fraction $(\boldsymbol{x})$ to account for the equilibrium between the bulk gas phase and the residual gas remained in the adsorbed phase (i.e., inside the MOF). Since the feed and operating conditions are fixed during the CSS operation for ideal VSA and PSA simulations, we assume the following variables, $y_{\text {feed }}, T_{\text {feed }}, P_{\text {high }}, P_{\text {low }}$, are constant.

Based on the material balance, the uptake difference of component $i$ in a MOF between the adsorption and desorption periods provides us with the information about amount of component $i$ in the bulk gas phase during the desorption period. For component $i$, we could formally defined the uptake difference as:

$$
\Delta q_{i} \equiv q_{a d s, i}-q_{d e s, i}
$$

Note that the uptake difference is not equivalent to the working capacity in that the uptake difference defined here accounts for different partial pres- 
sures of $\mathrm{SF}_{6}$ and $\mathrm{N}_{2}$ at the desorption period. Here, $q_{a d s, i}$ and $q_{d e s, i}$ are the adsorbed amount of component $i$ in a MOF at adsorption and desorption conditions, respectively.

Next, we define the sum of the uptake difference between the two periods of all components, which is equivalent to the total amount of gas in the bulk gas phase during the desorption period, as following:

$$
\Delta q_{t o t}=\sum_{j=1}^{2} \Delta q_{j}
$$

From this expression, we can define the $\mathrm{SF}_{6}$ purity $\left(x_{1}\right)$ as the ratio of amount of $\mathrm{SF}_{6}$ in the bulk gas phase $\left(\Delta q_{1}\right)$ and the total amount of all components in the bulk gas phase during the desorption period as in Eq. (17):

$$
x_{1}=\frac{\Delta q_{1}}{\Delta q_{t o t}}
$$

To express a single cycle operation, a function for the extract composition of $\mathrm{SF}_{6}\left(x_{1}\right)$ can be expressed as a function of the extract composition $(\boldsymbol{x})$ as follows:

$$
h_{1}(\boldsymbol{x}) \equiv \frac{f_{i s o, 1, a d s}-f_{i s o, 1, d e s}(\boldsymbol{x})}{f_{i s o, 1, a d s}-f_{i s o, 1, d e s}(\boldsymbol{x})+f_{i s o, 2, a d s}-f_{i s o, 2, d e s}(\boldsymbol{x})}
$$


Note that $f_{i s o, 1, a d s}$ and $f_{i s o, 2, a d s}$ are constants. To find the CSS condition (i.e., after repeated adsorption/desorption periods), we need to recursively call the function, $h(\boldsymbol{x})$ as in Eq. 19):

$$
x_{1}=h_{1}\left(h_{1}\left(\ldots h_{1}(\boldsymbol{x})\right)\right)=h_{1} \circ h_{1} \circ \ldots h_{1}(\boldsymbol{x})
$$

In the case for $\mathrm{SF}_{6}$ and $\mathrm{N}_{2}$, we need to consider two functions $\left(h_{1}\right.$ and $h_{2}$ ) simultaneously. For the multi-component case (where $n>2$ ), we should compute the mole fraction of all components using $n-1$ number of Eq. (19), namely $x_{i}$ for $i=1,2, \ldots, n$, simultaneously.

For all gas components, we can write down $H$ as follows:

$$
\boldsymbol{x}=H \circ H \circ \ldots H(\boldsymbol{x})
$$

To find the solution of Eq. (20), we employed the least-square optimization methods as implemented in the SciPy[32] with the formulation of Eq. (21):

$$
\begin{aligned}
& \min _{\boldsymbol{x}}\|\boldsymbol{x}-H(\boldsymbol{x})\|_{2}^{2} \\
& \text { s.t. } \sum_{i=1}^{n} x_{i}=100 \%
\end{aligned}
$$

Note that both VSA and PSA processes share the same equations for the composition of the extract stream produced during the desorption period. 
Jupyter Notebook with analyses code and data are available as part of the Supplementary Information.

\subsection{Energy Consumption}

The energy consumption to produce the desired product is directly related to the cost of the process operation. Energy consumption levels for the VSA and PSA were evaluated to compare the energy efficiency of these operations to produce $90 \%$ purity $\mathrm{SF}_{6}$. For the $\mathrm{SF}_{6}$ separation case, several modifications to the original method from Ga and co-workers 28] were made for both extract composition and energy consumption.

The key modification to the original method is the consideration of vacuum pump and compressor location (depending on the VSA and PSA). For example, different types of pressure driving equipment are required for VSA and PSA operations. Vacuum pump is required during the desorption period of the VSA operation while the compressor is required during the adsorption period of the PSA operation. Note that for the PSA operation, a compressor is located before the adsorption column so that the feed gas to the adsorption column is compressed, and then injected to the column. For the VSA operation, a vacuum pump is located after the column. The energy consumption required to produce a mole of $\mathrm{SF}_{6}$ was calculated using the Eq. (23) and Eq. (24), for vacuum pump and compressor, respectively. 


$$
\begin{gathered}
\frac{W_{v a c}}{x_{S F_{6}} \dot{n}_{\text {ext }}}=\frac{1}{x_{S F_{6}} \dot{n}_{\text {ext }}} \frac{\dot{n}_{\text {ext }} R T}{\eta_{\text {isen }}}\left(\left(\frac{P_{h}}{P_{l}}\right)^{\frac{\gamma-1}{\gamma}}-1\right) \\
=\frac{1}{x_{S F_{6}}} \frac{R T}{\eta_{\text {isen }}}\left(\left(\frac{P_{h}}{P_{l}}\right)^{\frac{\gamma-1}{\gamma}}-1\right)
\end{gathered}
$$

where,

$$
\begin{gathered}
P_{h}=P_{\text {feed }}, \\
P_{l}=P_{\text {vac }} \\
\frac{W_{\text {comp }}}{x_{S F_{6}} \dot{n}_{\text {ext }}}=\frac{1}{x_{S F_{6}} \dot{n}_{\text {ext }}} \frac{\dot{n}_{\text {feed }} R T}{\eta_{\text {isen }}}\left(\left(\frac{P_{h}}{P_{l}}\right)^{\frac{\gamma-1}{\gamma}}-1\right) \\
=\frac{1}{y_{\text {feed }}} \frac{R T}{\eta_{\text {isen }}}\left(\left(\frac{P_{h}}{P_{l}}\right)^{\frac{\gamma-1}{\gamma}}-1\right)
\end{gathered}
$$

where,

$$
\begin{aligned}
& P_{h}=P_{a d s}, \\
& P_{l}=P_{\text {feed }}
\end{aligned}
$$

In Eq. (23) and Eq. 24), $\gamma, \eta_{\text {isen }}, R$ and $T$ are isentropic expansion factor (dimensionless), isentropic efficiency (dimensionless), gas constant ( $\mathrm{J} / \mathrm{mol}$. $\mathrm{K}$ ) and temperature $(\mathrm{K})$, respectively. Using the ideal adsorption process formalism with $100 \%$ purity of raffinate component and conservation of mass $\left(x_{S F_{6}} \dot{n}_{e x t}=y_{f e e d} \dot{n}_{f e e d}\right)$, the extract flowrate terms are cancelled out in Eq. (23) and Eq. (24). The energy per separated $\mathrm{SF}_{6}$ defined here is independent of the process scale. Note that due to the compression (from compressor) and expansion (from vacuum pump) the column is operated at $313 \mathrm{~K}$ instead of $298 \mathrm{~K}$ for both PSA and VSA operations. 


\begin{tabular}{cccc}
\hline Decision variable & Symbol & Lower bound & Upper bound. \\
\hline Vacuum pressure & $P_{\text {vac }}$ & 0.001 & 0.316 \\
\hline
\end{tabular}

Table 1: Lower and upper bounds for optimizing VSA operation

\subsection{Optimization of VSA Operation}

The required energy to separate $\mathrm{SF}_{6}$ at the target purity (90\%) was evaluated for the VSA process. The optimal desorption pressure was found for each MOF by minimizing the vacuum pump energy input with the purity constraint (i.e., $90 \% \mathrm{SF}_{6}$ purity). Using the Eq. (6) - (22), the optimization problem can be expressed as Eq. (25) with decision variables and their upper/lower bounds listed in Table 1 .

$$
\begin{aligned}
& \min _{P_{v a c}} \frac{W_{v a c}}{x_{S F_{6}} \times \dot{n}_{\text {ext }}} \\
& \text { s.t. }
\end{aligned}
$$

\subsection{Optimization of Cascade PSA Operation}

For cascade PSA process, we defined $H_{c s s}(\cdot)$ as a solution of Eq. (21) and Eq. (22). Since the extract from the first train is used as an input to the compressor for the second train, and these two trains are in CSS, we need to solve for the extract compositions from two trains in series. From the feed composition, the extract composition of the first PSA train $\left(x_{1 s t}\right)$ was obtained with Eq. (26), and the purity value was used in the second PSA process, calculating the second PSA purity $\left(x_{2 n d}\right)$ with Eq. (27). 


\begin{tabular}{lccc}
\hline Decision variable & Symbol & Lower bound & Upper bound \\
\hline First PSA pressure & $P_{h, 1}$ & 2.5 & 30 \\
Second PSA pressure & $P_{h, 2}$ & 2.5 & 30 \\
\hline
\end{tabular}

Table 2: Decision variables and their bounds for the optimization of the cascade PSA operation.

$$
\begin{aligned}
& x_{1 s t}=H_{c s s}\left(y_{\text {feed }}, T_{\text {feed }}, P_{h, 1}, P_{\text {low }}\right) \\
& x_{2 n d}=H_{c s s}\left(x_{1 s t}, T_{\text {feed }}, P_{h, 2}, P_{\text {low }}\right)
\end{aligned}
$$

In these equations, the operating pressure employed for each PSA train is marked with $P_{h, 1}$ and $P_{h, 2}$ for the first and second train, respectively.

We compute the optimal combination of first and second train pressures that minimizes the energy consumption that satisfies the $90 \% \mathrm{SF}_{6}$ purity constraint. This optimization problem could be expressed using Eq. 28 with the decision variables and their bounds listed in Table 2 ,

$$
\min _{P_{h, 1}, P_{h, 2}} \frac{W_{c o m p, 1}+W_{c o m p, 2}}{x_{2 n d} \times \dot{n}_{e x t, 2}}
$$

s.t.

$$
x_{2 n d} \geq 90 \%
$$

Eq. (29) and Eq. (31) were used to calculate the energy consumption levels of the first and second PSA train $\left(W_{\text {comp }, 1}, W_{\text {comp }, 2}\right)$.

The key parameters are used as displayed in Figure 6: $\mathrm{SF}_{6}$ purity at the first and second PSA trains $\left(x_{1 s t}, x_{2 n d}\right)$, flowrates at feed and extract flows 
in the first and second PSA trains $\left(\dot{n}_{f e e d}, \dot{n}_{e x t, 1}, \dot{n}_{e x t, 2}\right)$. There are also $\mathrm{SF}_{6}$ composition in the feed flow $\left(y_{\text {feed }}\right)$, isentropic efficiency of the compressor $\left(\eta_{\text {isen }}\right)$, isentropic expansion factor $(\gamma)$, gas constant $(R)$, and temperature $(T)$.

$$
\begin{aligned}
& \frac{W_{\text {comp }, 1}}{x_{2 n d} \dot{n}_{\text {ext }, 2}}= \frac{1}{x_{2 n d} \dot{n}_{\text {ext }, 2}} \frac{\dot{n}_{\text {feed }} R T}{\eta_{\text {isen }}}\left(\frac{\gamma}{\gamma-1}\right)\left(\left(\frac{P_{h, 1}}{P_{\text {inlet }}}\right)^{\frac{\gamma-1}{\gamma}}-1\right) \\
&= \frac{1}{y_{\text {feed }}} \frac{R T}{\eta_{\text {isen }}}\left(\frac{\gamma}{\gamma-1}\right)\left(\left(\frac{P_{h, 1}}{P_{\text {inlet }}}\right)^{\frac{\gamma-1}{\gamma}}-1\right) \\
& \frac{W_{\text {comp }, 2}}{x_{2 n d} \dot{n}_{\text {ext }, 2}}= \frac{1}{x_{2 n d} \dot{n}_{\text {ext }, 2}} \frac{\dot{n}_{\text {ext }, 1} R T}{\eta_{\text {isen }}}\left(\frac{\gamma}{\gamma-1}\right)\left(\left(\frac{P_{h, 2}}{P_{\text {inlet }}}\right)^{\frac{\gamma-1}{\gamma}}-1\right) \\
&\left.=\frac{1}{x_{1 s t}} \frac{R T}{\eta_{\text {isen }}}\left(\frac{\gamma}{\gamma-1}\right)\left(\left(\frac{P_{h, 2}}{P_{\text {inlet }}}\right)^{\frac{\gamma-1}{\gamma}}-1\right)^{-1}\right) \\
& y_{\text {feed }, 1 s t} \dot{n}_{\text {feed }}=x_{1 s t} \dot{n}_{\text {ext }, 1} \\
&=x_{2 n d} \dot{n}_{\text {ext }, 2}
\end{aligned}
$$

Using the ideal adsorption process assumptions and the mass conservation of $\mathrm{SF}_{6}(33)$, the effect of flowrate could be removed from the equation, and the energy consumption could be expressed only as a function of each pressure $\left(P_{h, 1}\right.$ and $\left.P_{h, 2}\right)$ as Eq. (30) and Eq. (32). 


\section{Results and Discussion}

\subsection{High-throughput Computational Screening}
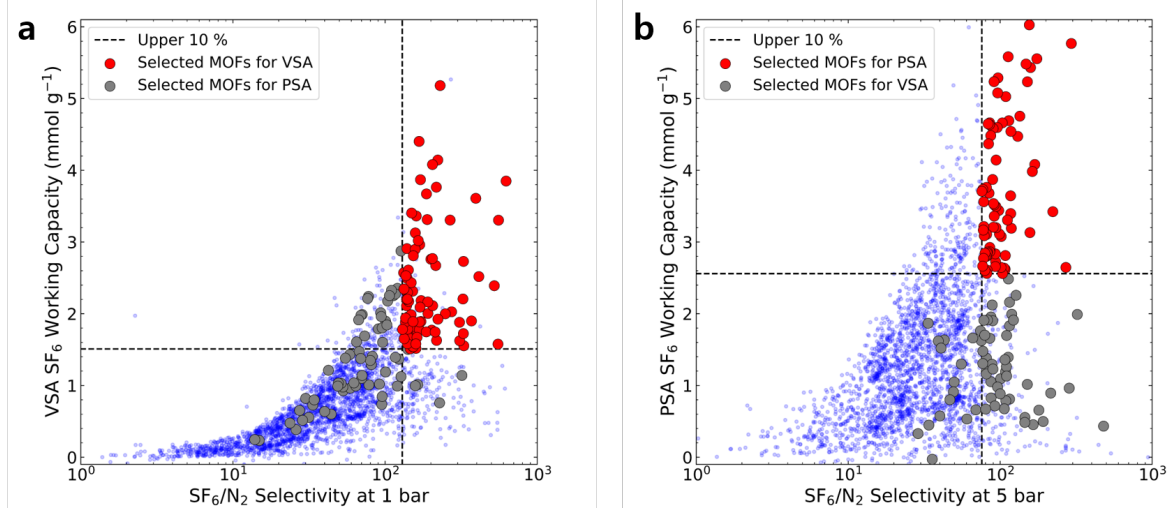

Figure 3: High-throughput computational screening (HTS) results for 2,890 MOFs. The

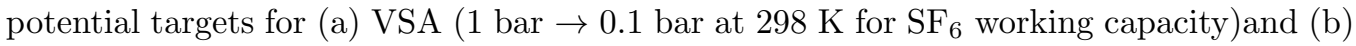
PSA ( 5 bar $\rightarrow 1$ bar at $298 \mathrm{~K}_{\text {for }} \mathrm{SF}_{6}$ working capacity). The criteria were upper $10 \%$ of both $\mathrm{SF}_{6}$ working capacity and $\mathrm{SF}_{6} / \mathrm{N}_{2}$ selectivity.

In order to identify high-performing MOFs for the separation, we used the performance metrics, such as selectivity and working capacity, that are commonly used in the literature. Figure 3 shows the high-throughput GCMC simulation results for 2,890 MOFs. From these results, we selected the top 10 \% MOFs based on the $\mathrm{SF}_{6} / \mathrm{N}_{2}$ selectivity and $\mathrm{SF}_{6}$ working capacity at VSA and PSA conditions. The top-performing MOFs for VSA operation have high $\mathrm{SF}_{6} / \mathrm{N}_{2}$ selectivity in range of 130 - 624 (at 1 bar), and the working capacity in the range of $1.5-5.2 \mathrm{mmol} / \mathrm{g}$. For PSA operation, the $\mathrm{SF}_{6} / \mathrm{N}_{2}$ selectivity is in the range of 76 - 293 (at 5 bar) while the $\mathrm{SF}_{6}$ working capacity is in the range of $2.7-6.4 \mathrm{mmol} / \mathrm{g}$. The number of top-performing MOFs for 
VSA and PSA conditions are 87 and 74, respectively, and these MOFs were further evaluated using the process-level simulation. Note that none of the MOFs in top $10 \%$ of VSA condition overlaps with the top $10 \%$ materials from PSA condition.

\subsection{Performance Evaluation based on Ideal PSA and VSA Simulations}
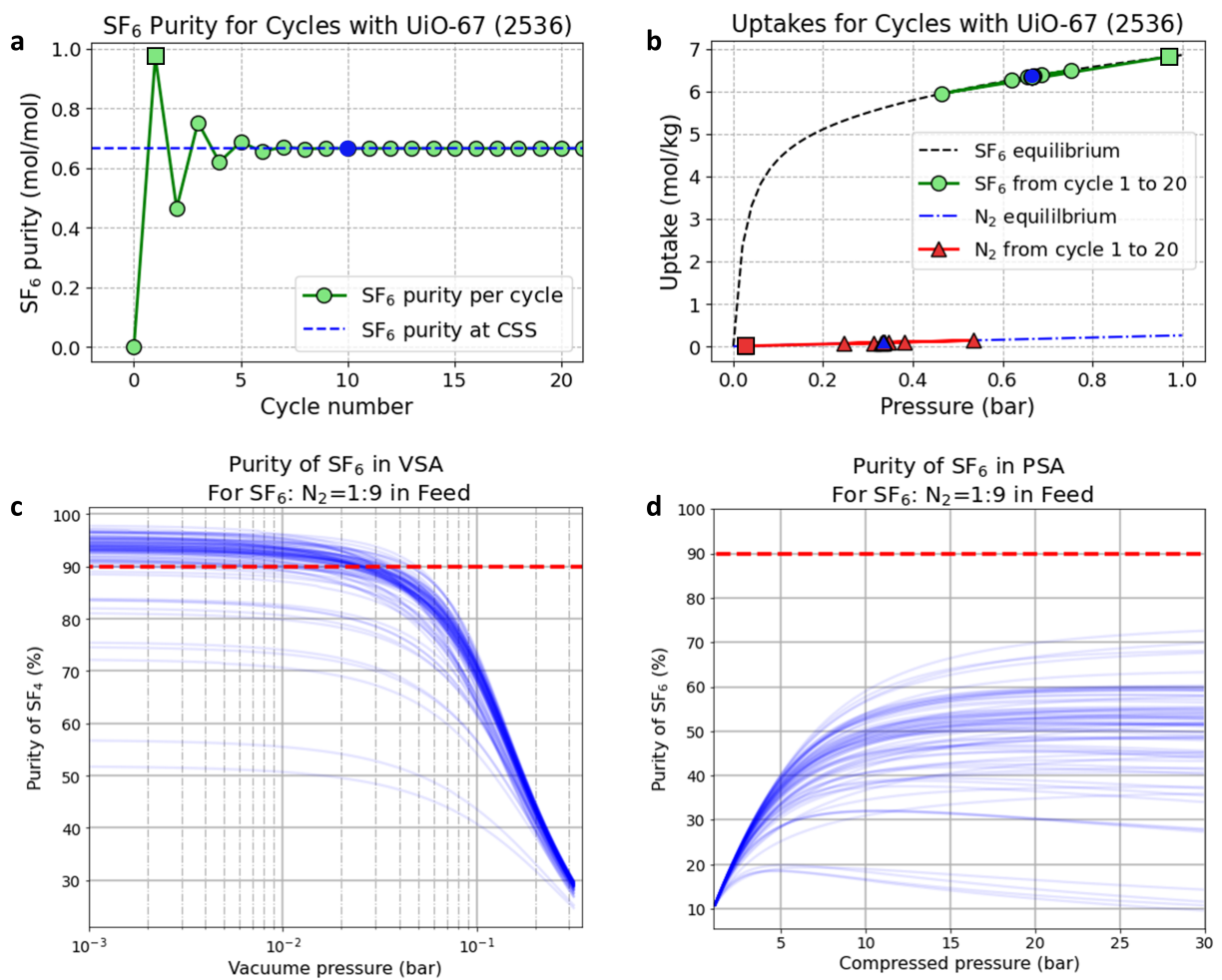

Figure 4: (a) SF6 purity computed for each cycle of the ideal PSA process with UiO-67 (Screening ID: 2536) (b) $\mathrm{SF}_{6}$ and $\mathrm{N}_{2}$ uptake varying for cycles with UiO-67, whose first and $0.1 \%$ CSS points are marked with square and blue markers, respectively (c) Ideal VSA and (d) Ideal PSA simulation results for the potential MOFs. SF 6 purity (\%) at the raffinate.

For practical purposes, the performance evaluation metrics used for the screening should, in principle, correlate with the performance of MOFs in 
the actual PSA and VSA processes. For binary gas separation cases, the selectivity and working capacity have been used frequently in the literature [33, 34, 35], but recent studies have shown that these commonly used metrics do not necessarily reflect the performance of MOFs at the process-level [36, 37, 38, 29. We adopted an ideal VSA/PSA process simulation method to quickly evaluate the performance of MOFs at the process-level. The ideal VSA or PSA process simulation that we employed in this work provides what would be an upper bound performance that a MOF could physically achieve.

Figure 4 (a) and (b) show an example of the ideal adsorption process simulation for a given MOF. Here, the bulk gas phase concentration (Figure 4 (a)) and the adsorbed phase uptakes (Figure 4 (b)) after the first cycle are labeled with square markers, and the data points corresponding to the earliest cycle that satisfies $0.1 \%$ CSS condition are colored in blue. At the first desorption step (i.e., end of the "first" cycle), the purity of $\mathrm{SF}_{6}$ is close to $100 \%$. This is because there is not much $\mathrm{N}_{2}$ adsorbed onto the MOF, and the extract initially contains highly concentrated $\mathrm{SF}_{6}$. However, as the system reaches the CSS, the bulk phase $\mathrm{SF}_{6}$ concentration (i.e., $\mathrm{SF}_{6}$ purity) decreases from the initial concentration and reaches a steady-state value.

The calculations were repeated at different desorption pressure (up to 0.001 bar) and adsorption pressure (up to 30 bar) for all 87 MOFs and 74 MOFs for VSA and PSA, respectively. The results of the ideal VSA and PSA simulations for MOFs are shown in Figure 4 (c) and (d). For the VSA process, we find that the $\mathrm{SF}_{6}$ purity increases as we apply more vacuum to the 
system. Some MOFs show larger increase in $\mathrm{SF}_{6}$ purity than the other MOFs as we pull more vacuum. Considering that a higher vacuum level requires more energy input to the VSA system, our results suggest that there could be a trade-off between the $\mathrm{SF}_{6}$ purity and the energy consumption.

Figure 4 (d) shows the results of the PSA process. In this operation, the compressed pressure is the manipulating variable for the operation. Similar to the VSA operation, we find that the higher (instead of lower vacuum in the VSA) compressed pressure typically yields a higher $\mathrm{SF}_{6}$ purity for the PSA process. However, we found the $\mathrm{SF}_{6}$ purity of a few MOFs starts to decrease as we further increase the compression pressure. This is likely due to the rate of $\mathrm{N}_{2}$ uptake swing increment is larger than that of $\mathrm{SF}_{6}$, which originates from the fact that the $\mathrm{SF}_{6}$ isotherms of these MOFs saturate early at relatively low pressure while the $\mathrm{N}_{2}$ isotherms gradually increase.

From the simulation results shown in Figure 4 (c) and (d), we found 74 out of 87 MOFs could achieve the $90 \% \mathrm{SF}_{6}$ purity for VSA. However, none of the MOFs was able to achieve the target purity with a single PSA train. This is striking because the selectivity and working capacity of the selected MOFs in each VSA and PSA have similar (Figure 3 and Supplementary Information Figure S1 to S3) values but some of the MOFs were able to meet the process target while the others were not able to do so.

We compared if the purity rankings of MOFs changes as we change the operating conditions for VSA and PSA. The Spearman's ranking correlation coefficients (SRCCs) were calculated between 0.001 bar and 0.1 bar for VSA 
and between 5 bar and 30 bar for PSA. We found the SRCC for VSA and PSA are high (0.872 for VSA and 0.918 for PSA) suggesting that the purity rankings of these MOFs do not change as we change the operating conditions (Supplementary Information Figure S4).

The codes for the above are available in:

https://github.com/sebyga/SF6-separation-VSA.git

\subsection{Energy Consumption: Ideal VSA Process}
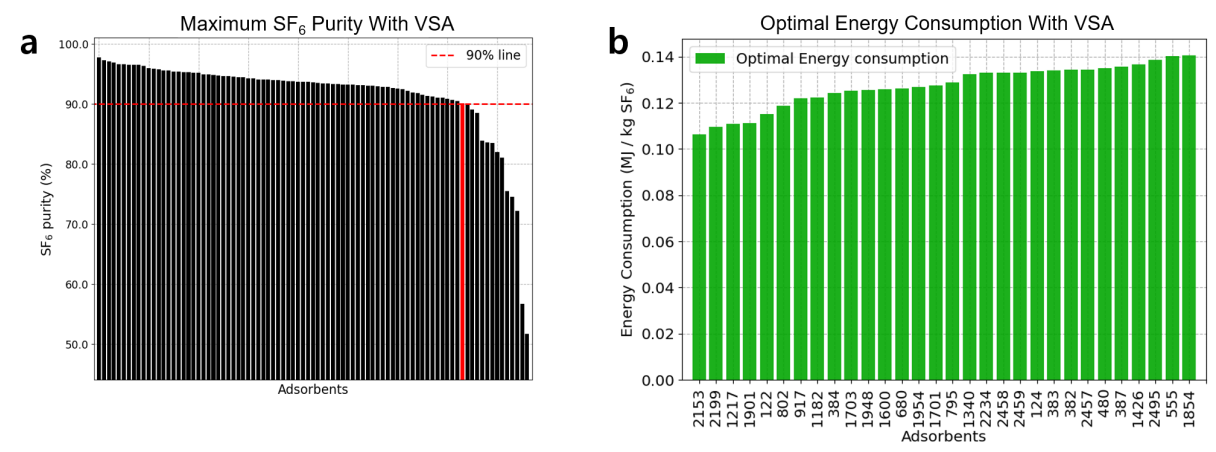

Figure 5: (a) Maximum $\mathrm{SF}_{6}$ purity of each adsorbents in an ideal VSA process with desorption pressure ranging 1 mbar to 0.316 bar (b) Energy consumption with optimal operation of VSA for the selected adsorbents having over $90 \%$ maximum $\mathrm{SF}_{6}$ purity

Figure 5 (a) shows the maximum $\mathrm{SF}_{6}$ purity that can be reached under the ideal VSA process. Here we find 74 out of 87 MOFs could reach beyond the $90 \%$ purity level. Since the $\mathrm{SF}_{6}$ purity is a continuous function with respect to the vacuum pressure, we could instead change the operating pressure to produce the $90 \%$ target purity while reducing the energy consumption of the vacuum pump. Figure 5 (b) shows the optimal energy consumption required to produce $90 \%$ of target $\mathrm{SF}_{6}$ purity under VSA condition. The 
energy consumption levels of different MOFs were computed and compared at their optimal pressure swing ranges. For top-performing MOFs, the range of energy consumption required to produce $90 \%$ purity level for the VSA is 0.10 - 0.14 MJ per $\mathrm{kg}$ of $\mathrm{SF}_{6}$. The detailed information about the top-performing MOFs are provided in Table S4 in the Supplementary Information.

\subsection{Cascade PSA Process}
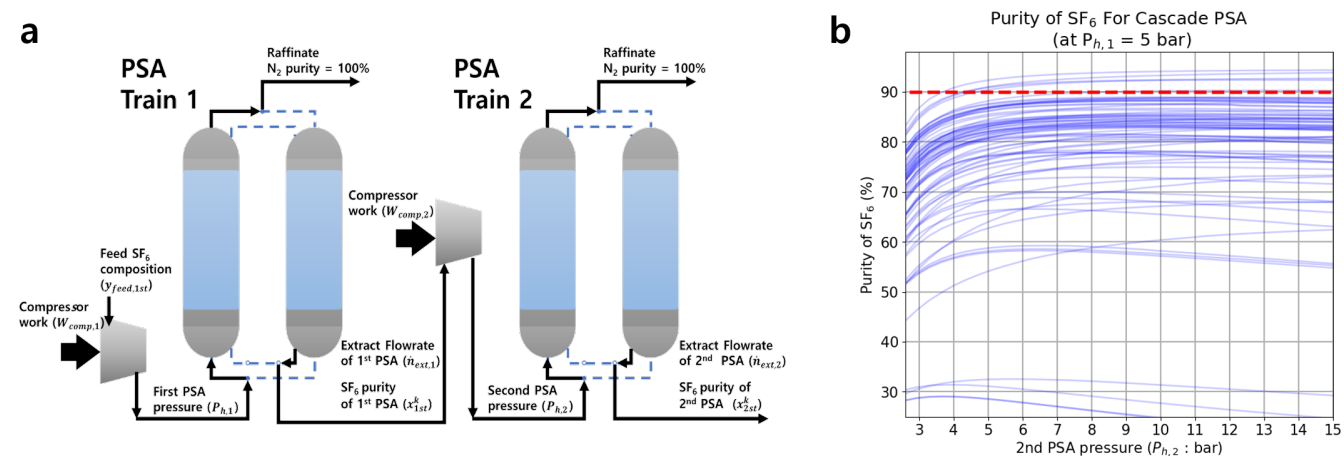

Figure 6: (a) Process flow diagram of the cascade PSA process and its key variables and (b) $\mathrm{SF}_{6}$ purity from $74 \mathrm{MOF}$ in the extract flow of the second PSA train with different 2nd train pressure

We found that none of the high-performing MOFs identified from the high-throughput computational screening were able to separate $\mathrm{SF}_{6}$ with 90\% purity with a single train PSA process while many of the MOFs were able to meet the target with VSA process. In order to achieve the target $\mathrm{SF}_{6}$ purity with PSA, we considered using two PSA trains in series (Figure 6 (a)) as an alternative strategy. The cascade configuration is advantageous because the configuration could be used achieve the target $\mathrm{SF}_{6}$ product purity. In this configuration, the extract flow from the first train enters the 
second train with slightly increased $\mathrm{SF}_{6}$ concentration, which allows the increased purity of the extract flow in the second train. Another more subtle (but important) advantage is that the cascade configuration could reduce the energy consumption per $\mathrm{kg}$ of $\mathrm{SF}_{6}$ by taking smaller pressure ratios of the two compressors. The optimal pressure combination that minimizes the energy consumption could be found using the equations (26) - (33).

We carried out the cascade PSA simulation on the 74 MOFs. We fixed the pressure of the first PSA train at 5 bar while varying the second PSA pressure $\left(P_{h, 2}\right)$ from 2.5 to 15 bar. The maximum $\mathrm{SF}_{6}$ purity values of the 74 MOFs were found using the cascade PSA configuration with a $10: 90 \mathrm{SF}_{6} / \mathrm{N}_{2}$ mixture in the feed flow at $313 \mathrm{~K}$ immediately after the first compressor. The heat exchanger was used with $298 \mathrm{~K}$ cooling water and a $15 \mathrm{~K}$ minimum temperature difference approach was used to remove the heat of compression within the compression system. The same approach was used for the feed flow from the first train to the second train with different feed flow composition to the second train. We explored both pressure directions by setting the first and second PSA pressures between 2.5 and 20 bar.

Figure 6 (b) shows the results of the cascade PSA process. The $\mathrm{SF}_{6}$ purity of the 74 MOFs are shown for a given range of the second PSA train pressure. Similar to the single train PSA, most of the curves exhibit the purity increase as the operating pressure increases. We also found few MOFs that show decrease in the $\mathrm{SF}_{6}$ purity with increasing pressure after a certain pressure point, indicating that there is a purity maximum with the cascade 
PSA configuration for some MOFs. Using this configuration, 36 out of 74 MOFs were able to meet the target $\mathrm{SF}_{6}$ purity level (90\%).

\subsection{Energy Consumption: Cascade PSA Process}
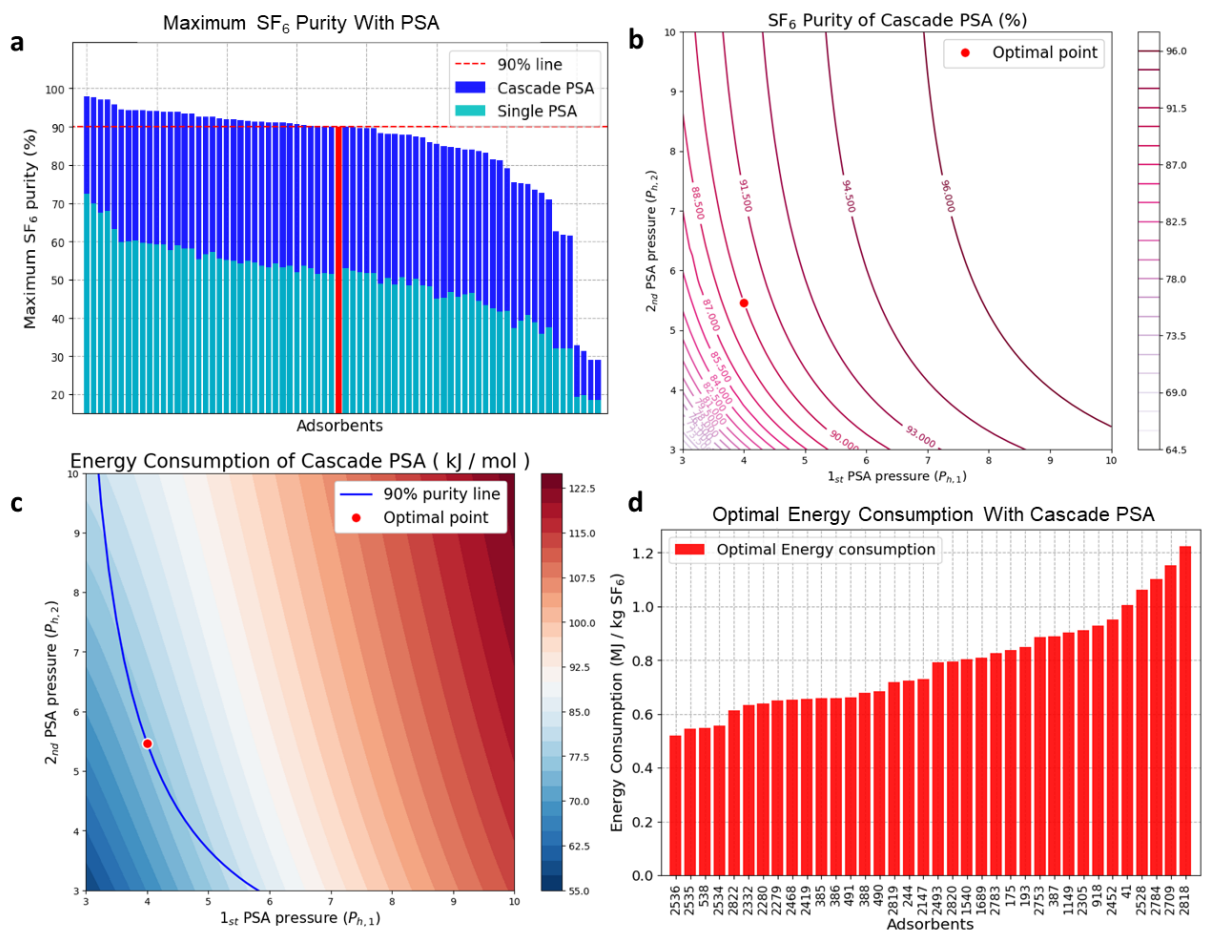

Figure 7: (a) Maximum $\mathrm{SF}_{6}$ purity achievable with different MOFs in a cascade PSA process (blue) and in a single PSA process (cyan). $90 \%$ of target purity is shown in red dotted line; (b) an example contour plot for UiO-67 with different combinations of PSA pressures and corresponding $\mathrm{SF}_{6}$ purity; (c) an energy consumption contour map of a cascade PSA for UiO-67 (screening ID: 2536) with varying pressure combinations with $90 \%$ purity line (blue line) and the optimal operating condition (red dot); (d) Energy consumption of the cascade PSA process with each optimal operation for 36 MOFs.

Figure 7 (a) shows the results from both single PSA and cascade PSA operations where the maximum purity values between the two operations are compared. We found that the cascade PSA operation leads to improved 
purity values compared to the single PSA operation. The average purity of the MOFs increased from $78.54 \%$ to $92.94 \%$. For all the MOFs that were not able to reach the $90 \%$ target purity with a single train PSA, we discovered some MOFs have the maximum purity values exceeding the target with the cascade PSA operation. We calculated the energy consumption of the cascade PSA operation for 36 MOFs that were able to achieve the $90 \%$ target purity level.

To illustrate how the optimization in the cascade PSA operates, the steps to find the optimal pressure combination that minimizes the energy consumption for UiO-67 (Screening ID: 2536) is shown in Figure 7(b) and (c). Figure 7 (b) shows the $\mathrm{SF}_{6}$ purity for varying pressure points. In the variable space, the feasible region is obtained based on the purity constraint in which the boundary is drawn with blue line as shown in Figure 7 (c). Among the feasible points in the region, the least energy consuming condition (i.e., pressure) is selected (red dot). The optimization results of all $36 \mathrm{MOF}$ are displayed in Figure 7 (d).

The codes for the above are available in:

https://github.com/sebyga/SF6-separation-PSA.git

3.6. Comparison between VSA and PSA with Different Isentropic Efficiencies

We compared the results of VSA and cascade PSA processes. Because the VSA process has a pressure driving equipment at the extract gas, less 

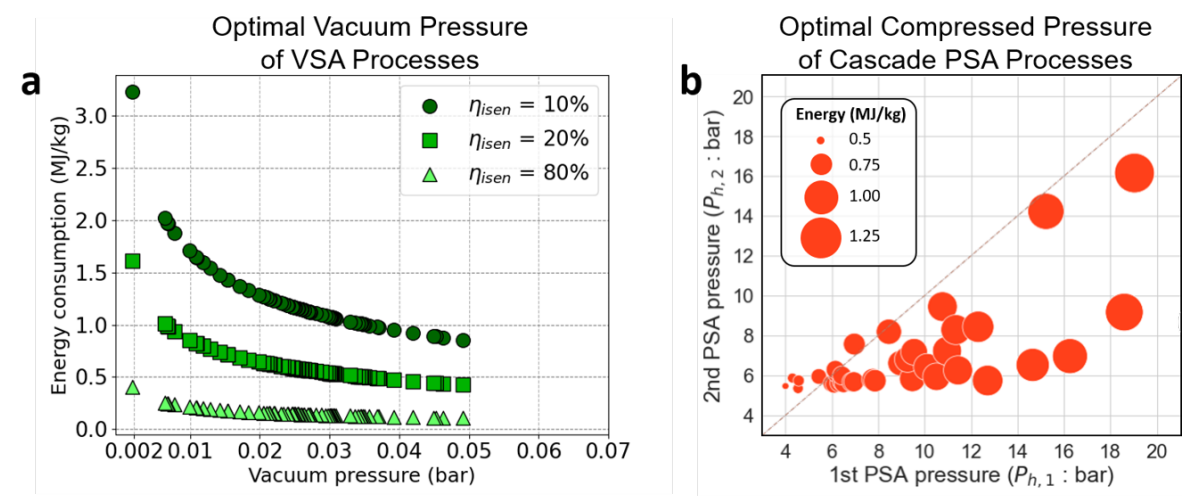

C

Energy Consumption for Different Vacuum Pump

Efficiencies (Top 3 Candidates)

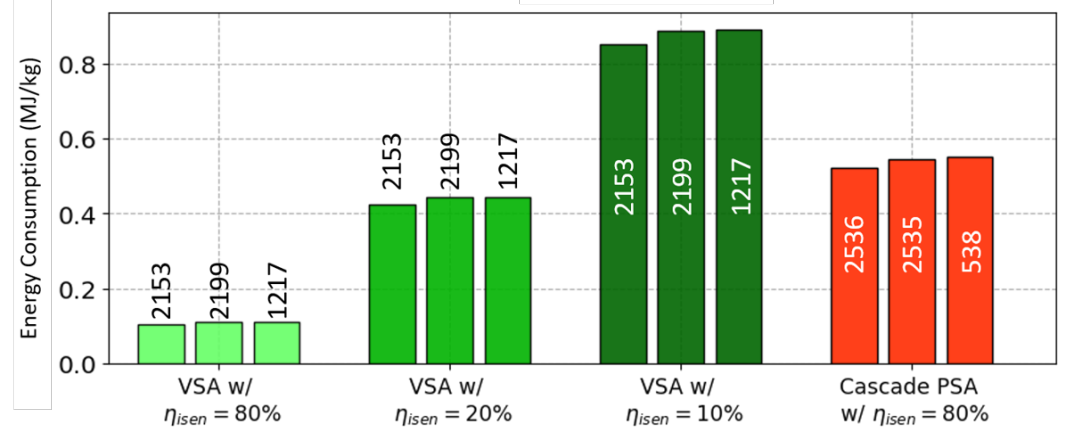

Figure 8: (a) Optimal vacuum pressure and energy consumption for 74 MOFs. 80, 20, $10 \%$ isentropic efficiency results are marked with light green triangles, normal green squares, and dark green circles, respectively; (b) Optimal compressed pressures and energy consumption for the first and the second PSA trains for 36 MOFs; (c) Comparison of the energy consumption values for different vacuum pump efficiencies.

amount of flowrate needs to be processed, which is advantageous when it comes to the energy consumption in the separation system. However, the use of strong vacuum is less preferable in the industrial application because the strong vacuum is known for its low energy efficiency [39, 40, 41]. This can be worse when the pressure ratio becomes higher. On the the contrary, the PSA process uses a compressor with less pressure ratio, which contributes its favorable application to various areas. Still, the compressor should deal with 
a large flowrate because it is located before the PSA column. This means the PSA process spends more energy for compressing the $\mathrm{N}_{2}$ than $\mathrm{SF}_{6}$.

Figure 8 (a) and (b) show the optimal operating pressures. As discussed previously, each MOF has a pressure point that minimizes the energy consumption with respect to the $90 \%$ purity constraint. Figure 8 (a) shows the optimal pressure is distributed between 2 mbar to 0.05 bar for all MOFs that could achieve the $90 \%$ purity target with $0.1-0.4 \mathrm{MJ}$ per $\mathrm{kg}$ of $\mathrm{SF}_{6}$. The optimal pressure values of the cascade PSA process are shown in Figure 8 (b). The size of each point reflects the degree of energy consumption of a MOF for a given pressure combination. While the pressure ratio for the vacuum pump (VSA) is between 15 and 200, the pressure ratio for the compressor (cascade PSA) is between 4 and 20. We can see that the VSA process requires higher pressure ratio for the operation, which indicates that the system has less isentropic efficiency.

To consider the effect of isentropic efficiency for the VSA process, we computed the energy consumption of the VSA process with different isentropic efficiency scenarios, and the results were compared with the cascade PSA results. Figure 8 (a) shows the results with 80, 20, $10 \%$ isentropic efficiencies. Three top-ranked MOFs with 80, 20, 10\% efficiency scenarios from the VSA process are displayed with the best performing MOFs from the cascade PSA process. In $80 \%$ and $20 \%$ scenarios, the energy required to separate $\mathrm{SF}_{6}$ with a VSA process is lower than that of the cascade PSA case. However, when the isentropic efficiency is assumed $10 \%$, the cascade PSA process outper- 
Table 3: Physical properties and their KPIs of Top 10 MOFs for optimized VSA condition.

\begin{tabular}{|c|c|c|c|c|c|c|c|c|}
\hline Screening ID & $\begin{array}{l}\text { LCD } \\
(\AA)\end{array}$ & $\begin{array}{l}\text { PLD } \\
(\AA)\end{array}$ & $\begin{array}{l}\mathrm{SF}_{6} \text { Heat of } \\
\text { Adsorption } \\
(\mathrm{kJ} / \mathrm{mol})\end{array}$ & $\begin{array}{l}\text { Surface } \\
\text { Area } \\
\left(\mathrm{m}^{2} / \mathrm{g}\right)\end{array}$ & $\begin{array}{l}\mathrm{SF}_{6} / \mathrm{N}_{2} \\
\text { Selectivity } \\
\text { (1 bar) }\end{array}$ & $\begin{array}{l}\mathrm{SF}_{6} \mathrm{WC} \\
(1-0.1 \mathrm{bar}) \\
(\mathrm{mmol} / \mathrm{g})\end{array}$ & $\begin{array}{l}\text { VSA Energy } \\
\text { Consumption } \\
\text { (MJ/kg) }\end{array}$ & References \\
\hline 2153 & 8.4 & 6.7 & 32.6 & 1313 & 325 & 2.2 & 0.1035 & Ding et al. 2015 42$]$ \\
\hline 2199 & 7.2 & 6.1 & 24 & 2121 & 413 & 2.5 & 0.1114 & Hasegawa et al. 200743 \\
\hline 1217 & 10.5 & 9.4 & 26.7 & 2217 & 222 & 4.1 & 0.1233 & Elsaidi et al. 201444 \\
\hline 1901 & 10.7 & 6.5 & 35.4 & 1124 & 309 & 1.6 & 0.1106 & Brunet et al. 201745 \\
\hline 122 & 9.7 & 6.9 & 27.3 & 1387 & 327 & 2.7 & 0.1102 & Zhang et al. 2011 46 \\
\hline 802 & 12.3 & 7.1 & 22.3 & 2363 & 394 & 3.6 & 0.1187 & Zhang et al. 201547 \\
\hline 917 & 14.6 & 7.3 & 28.3 & 1829 & 551 & 1.6 & 0.1218 & Vagin et al. 20084 \\
\hline 1182 & 14.8 & 8.2 & 16.8 & 3815 & 329 & 1.6 & 0.1223 & Ramirez et al. 2016 49 \\
\hline 384 & 7.7 & 6.8 & 25 & 1996 & 273 & 2 & 0.1252 & Hu et al. 2014 50 \\
\hline 1703 & 8.7 & 6.3 & 32.1 & 3687 & 521 & 2.4 & 0.1251 & Duan et al. 201151 \\
\hline
\end{tabular}

Table 4: Physical properties and their KPIs of Top 10 MOFs for optimized cascade PSA condition.

\begin{tabular}{|c|c|c|c|c|c|c|c|c|}
\hline Screening ID & $\begin{array}{l}\mathrm{LCD} \\
(\AA)\end{array}$ & $\begin{array}{l}\text { PLD } \\
(\AA)\end{array}$ & $\begin{array}{l}\mathrm{SF}_{6} \text { Heat of } \\
\text { Adsorption } \\
(\mathrm{kJ} / \mathrm{mol})\end{array}$ & $\begin{array}{l}\text { Surface } \\
\text { Area } \\
\left(\mathrm{m}^{2} / \mathrm{g}\right)\end{array}$ & $\begin{array}{l}\mathrm{SF}_{6} / \mathrm{N}_{2} \\
\text { Selectivity } \\
\text { ( } 5 \text { bar) }\end{array}$ & $\begin{array}{l}\mathrm{SF}_{6} \mathrm{WC} \\
(5-1 \mathrm{bar}) \\
(\mathrm{mmol} / \mathrm{g})\end{array}$ & $\begin{array}{l}\text { Cascade PSA Energy } \\
\text { Consumption } \\
(\mathrm{MJ} / \mathrm{kg})\end{array}$ & References \\
\hline 2536 & 13 & 6.7 & 31.7 & 3062 & 90 & 3.2 & 0.5211 & Hobday et al. 2016 52 \\
\hline 2535 & 13 & 6.7 & 25.3 & 3067 & 94 & 3.2 & 0.5452 & Hobday et al. 201652 \\
\hline 538 & 13.3 & 6.7 & 18.6 & 2358 & 82 & 2.6 & 0.5498 & Peterson et al. 201453 \\
\hline 2534 & 13 & 6.7 & 17 & 3058 & 98 & 3.4 & 0.5576 & Hobday et al. $2016 \sqrt[52]{5}$ \\
\hline 2822 & 13.2 & 6.6 & 43.6 & 2400 & 86 & 2.9 & 0.6125 & Peterson et al. 201453 \\
\hline 2332 & 10.6 & 9.6 & 27.6 & 2341 & 81 & 3.8 & 0.6328 & Park et al. 2011 54 \\
\hline 2280 & 11.7 & 6.1 & 20.5 & 5168 & 135 & 4.8 & 0.6381 & Pang et al. 201655 \\
\hline 2279 & 12.5 & 6 & 33.1 & 5212 & 158 & 5.4 & 0.6512 & Pang et al. 2016 55 \\
\hline 2468 & 9.2 & 6.5 & 21 & 3885 & 99 & 3.1 & 0.6544 & Burtch et al. 201356 \\
\hline 2419 & 10.9 & 6.5 & 25.8 & 3276 & 77 & 3.2 & 0.6568 & Wang et al. 2013 57 \\
\hline
\end{tabular}

forms the VSA process, showing lower required energy than that of the VSA process.

\subsection{High-Performing MOFs for $\mathrm{SF}_{6} / \mathrm{N}_{2}$ Separation}

We identified top-performing MOFs with low-energy consumption from both VSA and cascade PSA processes Table 3.7 and Table 3.7, (full version in the Supplementary Information Table S4). Based on the data, we found that the physical properties of high-performing MOFs are different between VSA and PSA processes. For example, MOFs that optimizes the performance of the VSA process have the LCD average value of 10.5 A while the MOFs that optimizes the cascade PSA cascade have the LCD average value of $12.0 \AA$. We also found that the process KPIs ( $\mathrm{SF}_{6}$ purity and energy consumption) 

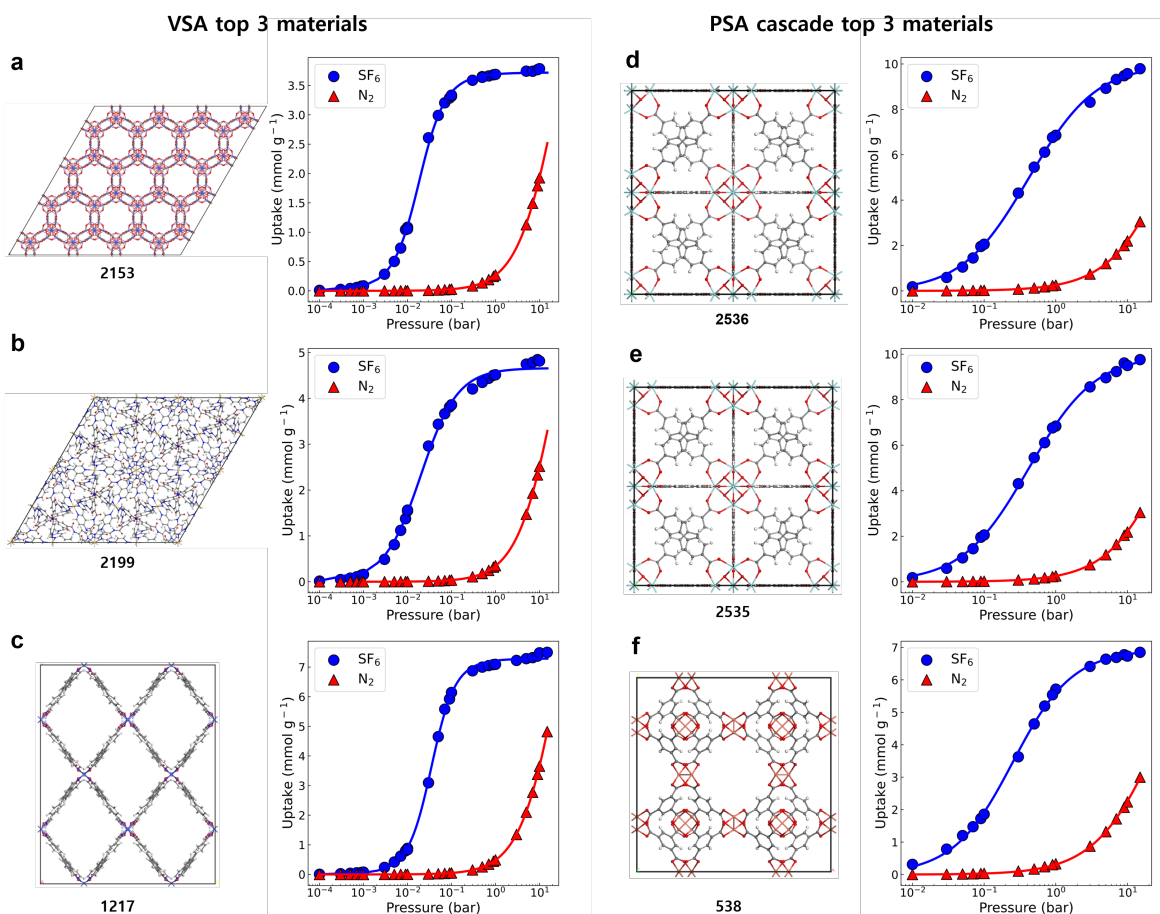

Figure 9: The structures and the adsorption isotherms for high performing top 3 materials in each VSA $(\mathrm{a}, \mathrm{b}, \mathrm{c})$ and cascade PSA $(\mathrm{d}, \mathrm{e}, \mathrm{f})$ simulation.

do not show any correlation with selectivity, and working capacities (see Supplementary Information Figures S2 and S3).

Figure 9 shows the molecular structure and the single component isotherms of the top 3 MOFs for VSA and cascade PSA. Among the best performing MOFs of the PSA case, UiO-67 (screening ID: 2536, 2535) and HKUST-1 (screeing ID: 538) are well-known MOFs in the literature that have been already validated for $\mathrm{SF}_{6} / \mathrm{N}_{2}$ separation [12, 11]. None of the top MOFs for VSA process have been experimentally tested for the application, which may be of a future study of interest. 
We found that top-performing MOFs from the cascade PSA process have different isotherm shapes than the VSA case. The best performing MOFs for VSA have the $\mathrm{SF}_{6}$ adsorption isotherm that saturates quickly in the pressure range of 0.01 bar to 2 bar whereas the best performing MOFs for cascade PSA have $\mathrm{SF}_{6}$ isotherms that gradually saturates near 10 bar. The maximum saturation loading is also different between the MOFs that are optimal for VSA and PSA. This is likely due to different structural properties of these MOFs and further research is required to draw the correlation between the structural properties of MOFs and their isotherm shape. 


\section{Conclusion}

In this work, more than 14,000 MOFs were computationally screened using a high-throughput, multi-scale evaluation method with two different process configurations (VSA and PSA). Hierarchical filtering and screening methods were used to find 87 and 74 potential target MOFs for subsequent VSA and PSA process simulations, respectively. We found that the 74 MOFs (out of 87 ) were able to achieve a $90 \%$ purity level but none of the MOFs selected for PSA were able to achieve the purity target. We explored the feasibility of achieving $90 \% \mathrm{SF}_{6}$ purity-level with a cascade PSA configuration, and found that 36 (out of the 74) MOFs were able to achieve $90 \% \mathrm{SF}_{6}$ purity-level with the cascade PSA configuration.

In terms of the energy consumption, we found top performing MOFs can produce $90 \%$ purity $\mathrm{SF}_{6}$ with $0.10-0.4$ and 0.5 - $1.4 \mathrm{MJ}$ per $\mathrm{kg}$ of $\mathrm{SF}_{6}$ for VSA and PSA, respectively. Furthermore, we revealed that the pressure ratio of VSA vacuum pump is higher than that of the PSA compressor (15 - 200 and 4 - 20 for VSA and PSA, respectively), which may impact the energy efficiency of the VSA process. We computed the energy consumption of MOFs with different isentropic efficiency values, and found that the cascade PSA process with $80 \%$ isentropic efficiency outperforms the VSA one with $10 \%$ isentropic efficiency. The results suggest that the isentropic efficiency of the vacuum pump must be considered when choosing between the VSA and PSA processes.

Finally, the structural properties of MOFs that optimizes the performance 
of the VSA process are different from the MOFs that optimizes the performance of the PSA process. $\left[\mathrm{H}_{2} \mathrm{DABCO}\right] \cdot\left[\mathrm{Co}\left(\mathrm{HPO}_{4}\right)\right.$ (bpdc)] (screening ID: 2153), [Cd(4-btapa) $\left.)_{2}\left(\mathrm{NO}_{3}\right)_{2}\right]$ (screening ID: 2199), and dia-7i-1-Co (screening ID: 1217) are the best performing MOFs for the VSA process, whereas UiO-67 (screening ID: 2535, 2536) and HKUST-1 (screning ID: 538) are the top-performing MOFs for the PSA process. None of the high-performing MOFs from the VSA process overlaps with the high-performing MOFs from the PSA process. A key factor that is responsible for the difference is the isotherm shape and the pressure point at which the $\mathrm{SF}_{6}$ uptake saturates. The saturation point of high pressure (over 1 bar) is favorable for the PSA process, and a relatively lower saturation point is required for the VSA process. The saturation point is likely determined by the structural properties of the MOFs. Top-performing MOFs for the PSA process have a larger LCD, PLD, pore volume, surface area, and void fraction than the top-performing VSA MOFs. Further research effort is necessary to draw the connection between the structural properties and the isotherm shape, and ultimately with the process KPIs.

\section{Declaration of Competing Interest}

The authors declare that they have no known competing financial interests or personal relationships that could have appeared to influence the work reported in this paper. 


\section{Acknowledgements}

Jaehoon Cha and Seongbin Ga contributed equally. This work was supported by the National Research Foundation of Korea (NRF) grant funded by the Korea government (MSTI) (No. 2020R1C1C101038311). The authors thank the computational time provided by KISTI (KSC-2019-CRE-0203).

\section{References}

[1] Global switchgear monitoring system market report 2019-2029, https://www.visiongain.com/report/ air-insulated-switchgear-market-2020/, accessed: 2020-02-28.

[2] J. Rogelj, M. Den Elzen, N. Höhne, T. Fransen, H. Fekete, H. Winkler, R. Schaeffer, F. Sha, K. Riahi, M. Meinshausen, Paris agreement climate proposals need a boost to keep warming well below 2 c, Nature 534 (7609) (2016) 631-639.

[3] P. G. Simmonds, M. Rigby, A. J. Manning, S. Park, K. M. Stanley, A. McCulloch, S. Henne, F. Graziosi, M. Maione, J. Arduini, et al., The increasing atmospheric burden of the greenhouse gas sulfur hexafluoride (sf 6), Atmospheric Chemistry and Physics 20 (12) (2020) 7271-7290.

[4] K. Shiojiri, Y. Yanagisawa, A. Yamasaki, F. Kiyono, Separation of fgases (hfc-134a and sf6) from gaseous mixtures with nitrogen by surface diffusion through a porous vycor glass membrane, Journal of membrane science 282 (1-2) (2006) 442-449. 
[5] A. Wolińska-Grabczyk, A. Jankowski, R. Sekuła, B. Kruczek, Separation of sf6 from binary mixtures with n2 using commercial poly (4-methyl-1pentene) films, Separation Science and Technology 46 (8) (2011) 12311240.

[6] J.-W. Choi, S. Lee, B. An, S.-B. Kim, S.-H. Lee, Separation of sulfur hexafluoride from a nitrogen/sulfur hexafluoride mixture using a polymer hollow fiber membrane, Water, Air, \& Soil Pollution 225 (2) (2014) 1807.

[7] D. Alezi, Y. Belmabkhout, M. Suyetin, P. M. Bhatt, Ł. J. Weselinski, V. Solovyeva, K. Adil, I. Spanopoulos, P. N. Trikalitis, A.-H. Emwas, et al., Mof crystal chemistry paving the way to gas storage needs: aluminum-based soc-mof for ch4, o2, and co2 storage, Journal of the American Chemical Society 137 (41) (2015) 13308-13318.

[8] F. X. L. i Xamena, A. Abad, A. Corma, H. Garcia, Mofs as catalysts: Activity, reusability and shape-selectivity of a pd-containing mof, Journal of Catalysis 250 (2) (2007) 294-298.

[9] Y. Cui, R. Song, J. Yu, M. Liu, Z. Wang, C. Wu, Y. Yang, Z. Wang, B. Chen, G. Qian, Dual-emitting mof dye composite for ratiometric temperature sensing, Advanced Materials 27 (8) (2015) 1420-1425.

[10] I. Senkovska, E. Barea, J. A. R. Navarro, S. Kaskel, Adsorptive capturing and storing greenhouse gases such as sulfur hexafluoride and carbon 
tetrafluoride using metal-organic frameworks, Microporous and mesoporous materials 156 (2012) 115-120.

[11] C. Y. Chuah, K. Goh, T.-H. Bae, Hierarchically structured hkust-1 nanocrystals for enhanced sf6 capture and recovery, The Journal of Physical Chemistry C 121 (12) (2017) 6748-6755.

[12] M.-B. Kim, T.-H. Kim, T.-U. Yoon, J. H. Kang, J.-H. Kim, Y.-S. Bae, Efficient sf6/n2 separation at high pressures using a zirconium-based mesoporous metal-organic framework, Journal of Industrial and Engineering Chemistry 84 (2020) 179-184.

[13] A. Raza, A. Sturluson, C. M. Simon, X. Fern, Message passing neural networks for partial charge assignment to metal-organic frameworks, The Journal of Physical Chemistry C 124 (35) (2020) 19070-19082.

[14] D. Ongari, L. Talirz, B. Smit, Too many materials and too many applications: An experimental problem waiting for a computational solution, ACS central science 6 (11) (2020) 1890-1900.

[15] Y. He, W. Zhou, G. Qian, B. Chen, Methane storage in metal-organic frameworks, Chemical Society Reviews 43 (16) (2014) 5657-5678.

[16] S. S. Kaye, A. Dailly, O. M. Yaghi, J. R. Long, Impact of preparation and handling on the hydrogen storage properties of zn4o (1, 4benzenedicarboxylate) 3 (mof-5), Journal of the American Chemical Society 129 (46) (2007) 14176-14177. 
[17] R. B. Getman, Y.-S. Bae, C. E. Wilmer, R. Q. Snurr, Review and analysis of molecular simulations of methane, hydrogen, and acetylene storage in metal-organic frameworks, Chemical reviews 112 (2) (2012) 703-723.

[18] B. J. Bucior, N. S. Bobbitt, T. Islamoglu, S. Goswami, A. Gopalan, T. Yildirim, O. K. Farha, N. Bagheri, R. Q. Snurr, Energy-based descriptors to rapidly predict hydrogen storage in metal-organic frameworks, Molecular Systems Design \& Engineering 4 (1) (2019) 162-174.

[19] H. Wang, X. Dong, J. Lin, S. J. Teat, S. Jensen, J. Cure, E. V. Alexandrov, Q. Xia, K. Tan, Q. Wang, et al., Topologically guided tuning of zr-mof pore structures for highly selective separation of c6 alkane isomers, Nature communications 9 (1) (2018) 1-11.

[20] E. A. Dolgopolova, A. M. Rice, C. R. Martin, N. B. Shustova, Photochemistry and photophysics of mofs: steps towards mof-based sensing enhancements, Chemical Society Reviews 47 (13) (2018) 4710-4728.

[21] Y. G. Chung, E. Haldoupis, B. J. Bucior, M. Haranczyk, S. Lee, H. Zhang, K. D. Vogiatzis, M. Milisavljevic, S. Ling, J. S. Camp, et al., Advances, updates, and analytics for the computation-ready, experimental metal-organic framework database: Core mof 2019, Journal of Chemical \& Engineering Data 64 (12) (2019) 5985-5998.

[22] D. Dubbeldam, S. Calero, D. E. Ellis, R. Q. Snurr, Raspa: molecular 
simulation software for adsorption and diffusion in flexible nanoporous materials, Molecular Simulation 42 (2) (2016) 81-101.

[23] S. L. Mayo, B. D. Olafson, W. A. Goddard, A generic force field for molecular simulations, J. Phys. Chem 94 (26) (1990) 8897-8909.

[24] C. Casewit, K. Colwell, A. Rappe, Application of a universal force field to organic molecules, Journal of the American chemical society 114 (25) (1992) 10035-10046.

[25] D. Dellis, J. Samios, Molecular force field investigation for sulfur hexafluoride: A computer simulation study, Fluid phase equilibria 291 (1) (2010) 81-89.

[26] B. L. Eggimann, A. J. Sunnarborg, H. D. Stern, A. P. Bliss, J. I. Siepmann, An online parameter and property database for the trappe force field, Molecular Simulation 40 (1-3) (2014) 101-105.

[27] B. Widom, Some topics in the theory of fluids, The Journal of Chemical Physics 39 (11) (1963) 2808-2812.

[28] S. Ga, H. Jang, J. H. Lee, New performance indicators for adsorbent evaluation derived from a reduced order model of an idealized psa process for co2 capture, Computers \& Chemical Engineering 102 (2017) $188-212$.

[29] S. Ga, S. Lee, J. Kim, J. H. Lee, Isotherm parameter library and evalu- 
ation software for co2 capture adsorbents, Computers \& Chemical Engineering 143 (2020) 107105.

[30] C. M. Simon, B. Smit, M. Haranczyk, pyiast: Ideal adsorbed solution theory (iast) python package, Computer Physics Communications 200 (2016) 364-380.

[31] A. L. Myers, J. M. Prausnitz, Thermodynamics of mixed-gas adsorption, AIChE journal 11 (1) (1965) 121-127.

[32] P. Virtanen, R. Gommers, T. E. Oliphant, M. Haberland, T. Reddy, D. Cournapeau, E. Burovski, P. Peterson, W. Weckesser, J. Bright, S. J. van der Walt, M. Brett, J. Wilson, K. J. Millman, N. Mayorov, A. R. J. Nelson, E. Jones, R. Kern, E. Larson, C. J. Carey, İ. Polat, Y. Feng, E. W. Moore, J. VanderPlas, D. Laxalde, J. Perktold, R. Cimrman, I. Henriksen, E. A. Quintero, C. R. Harris, A. M. Archibald, A. H. Ribeiro, F. Pedregosa, P. van Mulbregt, SciPy 1.0 Contributors, SciPy 1.0: Fundamental Algorithms for Scientific Computing in Python, Nature Methods 17 (2020) 261-272. doi:10.1038/s41592-019-0686-2.

[33] D. Saha, Z. Bao, F. Jia, S. Deng, Adsorption of co2, ch4, n2o, and n2 on mof-5, mof-177, and zeolite 5a, Environmental science \& technology 44 (5) (2010) 1820-1826.

[34] B. Yuan, X. Wu, Y. Chen, J. Huang, H. Luo, S. Deng, Adsorption of co2, ch4, and n2 on ordered mesoporous carbon: approach for green- 
house gases capture and biogas upgrading, Environmental science \& technology 47 (10) (2013) 5474-5480.

[35] S. Salehi, M. Anbia, F. Razavi, Improving co2/ch4 and co2/n2 adsorptive selectivity of cu-btc and mof-derived nanoporous carbon by modification with nitrogen-containing groups, Environmental Progress \& Sustainable Energy 39 (1) (2020) 13302.

[36] K. T. Leperi, D. Yancy-Caballero, R. Q. Snurr, F. You, 110th anniversary: Surrogate models based on artificial neural networks to simulate and optimize pressure swing adsorption cycles for co2 capture, Industrial \& Engineering Chemistry Research 58 (39) (2019) 18241-18252.

[37] K. N. Pai, V. Prasad, A. Rajendran, Generalized, adsorbent-agnostic, artificial neural network framework for rapid simulation, optimization, and adsorbent screening of adsorption processes, Industrial \& Engineering Chemistry Research 59 (38) (2020) 16730-16740.

[38] T. D. Burns, K. N. Pai, S. G. Subraveti, S. P. Collins, M. Krykunov, A. Rajendran, T. K. Woo, Prediction of mof performance in vacuum swing adsorption systems for postcombustion co2 capture based on integrated molecular simulations, process optimizations, and machine learning models, Environmental science \& technology 54 (7) (2020) 45364544 .

[39] D. Xu, P. Xiao, J. Zhang, G. Li, G. Xiao, P. A. Webley, Y. Zhai, Effects 
of water vapour on co2 capture with vacuum swing adsorption using activated carbon, Chemical Engineering Journal 230 (2013) 64-72.

[40] S. Krishnamurthy, V. R. Rao, S. Guntuka, P. Sharratt, R. Haghpanah, A. Rajendran, M. Amanullah, I. A. Karimi, S. Farooq, Co2 capture from dry flue gas by vacuum swing adsorption: a pilot plant study, AIChE Journal 60 (5) (2014) 1830-1842.

[41] R. Zhao, S. Deng, L. Zhao, Y. Zhao, S. Li, Y. Zhang, Z. Yu, Experimental study and energy-efficiency evaluation of a 4-step pressure-vacuum swing adsorption (pvsa) for co2 capture, Energy Conversion and Management 151 (2017) 179-189.

[42] Q.-R. Ding, L.-M. Li, L. Zhang, J. Zhang, Multiarylpolycarboxylatemediated hybrid cobalt phosphate frameworks with supramolecular zeolitic topology and unusual i2o2 connectivity, Inorganic chemistry 54 (4) (2015) 1209-1211.

[43] S. Hasegawa, S. Horike, R. Matsuda, S. Furukawa, K. Mochizuki, Y. Kinoshita, S. Kitagawa, Three-dimensional porous coordination polymer functionalized with amide groups based on tridentate ligand: selective sorption and catalysis, Journal of the American Chemical Society 129 (9) (2007) 2607-2614.

[44] S. K. Elsaidi, M. H. Mohamed, L. Wojtas, A. Chanthapally, T. Pham, B. Space, J. J. Vittal, M. J. Zaworotko, Putting the squeeze on ch4 and 
co2 through control over interpenetration in diamondoid nets, Journal of the American Chemical Society 136 (13) (2014) 5072-5077.

[45] G. Brunet, D. A. Safin, K. Robeyns, G. A. Facey, I. Korobkov, Y. Filinchuk, M. Murugesu, Confinement effects of a crystalline sponge on ferrocene and ferrocene carboxaldehyde, Chemical Communications 53 (41) (2017) 5645-5648.

[46] Z. Zhang, L. Wojtas, M. J. Zaworotko, Consequences of partial flexibility in 1, 3-benzenedicarboxylate linkers: Kagome lattice and nbo supramolecular isomers from complexation of a bulky 1, 3benzenedicarboxylate to $\mathrm{cu}$ (ii) paddlewheel moieties, Crystal growth \& design 11 (5) (2011) 1441-1445.

[47] S.-Y. Zhang, D. Li, D. Guo, H. Zhang, W. Shi, P. Cheng, L. Wojtas, M. J. Zaworotko, Synthesis of a chiral crystal form of mof-5, cmof-5, by chiral induction, Journal of the American Chemical Society 137 (49) (2015) 15406-15409.

[48] S. Vagin, A. Ott, H.-C. Weiss, A. Karbach, D. Volkmer, B. Rieger, Metal-organic frameworks (mofs) composed of (triptycenedicarboxylato) zinc (2008).

[49] J. R. Ramirez, H. Yang, C. M. Kane, A. N. Ley, K. T. Holman, Reproducible synthesis and high porosity of mer-zn (im) 2 (zif-10): exploita- 
tion of an apparent double-eight ring template, Journal of the American Chemical Society 138 (37) (2016) 12017-12020.

[50] Z. Hu, K. Tan, W. P. Lustig, H. Wang, Y. Zhao, C. Zheng, D. Banerjee, T. J. Emge, Y. J. Chabal, J. Li, Effective sensing of rdx via instant and selective detection of ketone vapors, Chemical Science 5 (12) (2014) 4873-4877.

[51] J. Duan, J. Bai, B. Zheng, Y. Li, W. Ren, Controlling the shifting degree of interpenetrated metal-organic frameworks by modulator and temperature and their hydrogen adsorption properties, Chemical Communications 47 (9) (2011) 2556-2558.

[52] C. L. Hobday, R. J. Marshall, C. F. Murphie, J. Sotelo, T. Richards, D. R. Allan, T. Düren, F.-X. Coudert, R. S. Forgan, C. A. Morrison, et al., A computational and experimental approach linking disorder, high-pressure behavior, and mechanical properties in uio frameworks, Angewandte Chemie 128 (7) (2016) 2447-2451.

[53] V. K. Peterson, P. D. Southon, G. J. Halder, D. J. Price, J. J. Bevitt, C. J. Kepert, Guest adsorption in the nanoporous metal-organic framework cu3 (1, 3, 5-benzenetricarboxylate) 2: combined in situ x-ray diffraction and vapor sorption, Chemistry of Materials 26 (16) (2014) 4712-4723.

[54] H. J. Park, D.-W. Lim, W. S. Yang, T.-R. Oh, M. P. Suh, A highly 
porous metal-organic framework: Structural transformations of a guestfree mof depending on activation method and temperature, ChemistryA European Journal 17 (26) (2011) 7251-7260.

[55] J. Pang, C. Liu, Y. Huang, M. Wu, F. Jiang, D. Yuan, F. Hu, K. Su, G. Liu, M. Hong, Visualizing the dynamics of temperature-and solvent-responsive soft crystals, Angewandte Chemie International Edition 55 (26) (2016) 7478-7482.

[56] N. C. Burtch, H. Jasuja, D. Dubbeldam, K. S. Walton, Molecular-level insight into unusual low pressure co2 affinity in pillared metal-organic frameworks, Journal of the American Chemical Society 135 (19) (2013) $7172-7180$.

[57] Z. Wang, B. Zheng, H. Liu, X. Lin, X. Yu, P. Yi, R. Yun, High-capacity gas storage by a microporous oxalamide-functionalized nbo-type metal-organic framework, Crystal growth \& design 13 (11) (2013) 5001-5006. 